\title{
Computational Methods for Solving Linear Fuzzy Volterra Integral Equation
}

\author{
Jihan Hamaydi and Naji Qatanani \\ Department of Mathematics, An-Najah National University, Nablus, State of Palestine \\ Correspondence should be addressed to Naji Qatanani; nqatanani@najah.edu
}

Received 21 February 2017; Accepted 18 April 2017; Published 28 May 2017

Academic Editor: Mehmet Sezer

Copyright (c) 2017 Jihan Hamaydi and Naji Qatanani. This is an open access article distributed under the Creative Commons Attribution License, which permits unrestricted use, distribution, and reproduction in any medium, provided the original work is properly cited.

\begin{abstract}
Two numerical schemes, namely, the Taylor expansion and the variational iteration methods, have been implemented to give an approximate solution of the fuzzy linear Volterra integral equation of the second kind. To display the validity and applicability of the numerical methods, one illustrative example with known exact solution is presented. Numerical results show that the convergence and accuracy of these methods were in a good agreement with the exact solution. However, according to comparison of these methods, we conclude that the variational iteration method provides more accurate results.
\end{abstract}

\section{Introduction}

Fuzzy integral equations of the second kind have attracted the attention of many scientists and researchers in recent years. These equations appear frequently in fuzzy control, fuzzy finance, approximate reasoning, and economic systems [1]. The concept of fuzzy sets was originally introduced by Zadeh [2] and led to the definition of fuzzy numbers and its implementation in fuzzy control [3] and approximate reasoning problems [4]. Dubois and Prade [5] were the first to introduce the concept of fuzzy functions. Alternative approaches were later suggested by Goetschel and Voxman [6], Kaleva [7], Nanda [8], and others.

In recent years, numerous methods have been proposed for solving Volterra integral equations [9]. Tricomi [10] was the first to introduce the successive approximations method for nonlinear integral equations. Liao [11] employed the homotopy analysis method to solve nonlinear problems and then it has been applied by Abbasbandy [12] to solve fuzzy Volterra integral equations of the second kind. Babolian et al. [13] have used the orthogonal triangular functions as a direct method for numerically solving integral equations system. Jafarian et al. [14] have solved systems of linear integral equations by using Legendre wavelets. Kanwal and Liu [15] implemented the Taylor expansion approach for solving integral equations while $\mathrm{Xu}$ [16] solved integral equations by the variational iteration method. In addition, Amawi and Qatanani $[17,18]$ have investigated the analytical and numerical treatment of fuzzy linear Fredholm integral equation. Hamaydi [19] has used various analytical and numerical methods to solve fuzzy Volterra integral equations.

The paper is organized as follows: in Section 2, fuzzy Volterra integral equation of the second kind is introduced. The Taylor expansion method used to approximate solution of fuzzy Volterra integral equation is addressed in Section 3. In Section 4, we present the variational iteration method (VIM) which provides a sequence of functions that converges to the exact solution to the problem.

The proposed methods are implemented using a numerical example with known exact solution by applying the MAPLE software in Section 5. Conclusions are given in Section 6.

\section{Fuzzy Volterra Integral Equation}

A standard form of the Volterra integral equation of the second kind is given by [14]

$$
u(x)=f(x)+\lambda \int_{a}^{x} k(x, t) u(t) d t,
$$


where $\lambda$ is a positive parameter, $k(x, t)$ is an arbitrary function called the kernel of the integral equation defined over square $G:[a, b] *[a, b], k(x, t)=0, a \leq t, x \leq b, t>x$, and $f(x)$ is a given function of $x \in[a, b]$. If $f(x)$ is a fuzzy function, then (1) is called a fuzzy Volterra integral equation of the second kind.

Definition 1 (see [20]). The second kind fuzzy Volterra integral equations system is of the following form:

$$
u_{i}(x)=f_{i}(x)+\sum_{j=1}^{m} \lambda_{i j} \int_{a}^{x} k_{i j}(x, t) u_{j}(t) d t
$$

where $a \leq t \leq x \leq b$ and $\lambda_{i j} \neq 0$ (for $i, j=1,2$, $3, \ldots, m)$ are real constants. Moreover, in system (2), the fuzzy function $f_{i}(x)$ and kernel $k_{i j}(x, t)$ are given and assumed to be sufficiently differentiable with respect to all their arguments on the interval $a \leq t, x \leq b$, and we assume that the kernel function $k_{i j}(x, t) \in G:[a, b] *[a, b]$, and $u_{j}(x)=$ $\left[u_{1}, u_{2}, \ldots, u_{m}\right]^{t}$ is the solution to be determined. Now let $\left(f_{i}(x, \alpha), \bar{f}_{i}(x, \alpha)\right)$ and $\left(\underline{u}_{i}(x, \alpha), \bar{u}_{i}(x, \alpha)\right), 0 \leq \alpha \leq 1, x \in$ $[\bar{a}, b]$, be the parametric forms of $f_{i}(x)$ and $u_{i}(x)$, respectively; then the parametric forms of the fuzzy Volterra integral equations system are as follows:

$$
\begin{aligned}
& \underline{u}_{i}(x, \alpha)=\underline{f_{i}}(x, \alpha)+\sum_{j=1}^{m}\left(\lambda_{i j} \int_{a}^{x} \underline{g}_{i j}(t, \alpha) d t\right), \\
& \bar{u}_{i}(x, \alpha)=\bar{f}_{i}(x, \alpha)+\sum_{j=1}^{m}\left(\lambda_{i j} \int_{a}^{x} \bar{g}_{i j}(t, \alpha) d t\right), \\
& i=1,2, \ldots, m,
\end{aligned}
$$

where

$$
\begin{aligned}
& \bar{g}_{i j}(t, \alpha)= \begin{cases}k_{i j}(x, t) \bar{u}_{j}(t, \alpha), & k_{i j}(x, t) \geq 0 \\
k_{i j}(x, t) \underline{u}_{j}(t, \alpha), & k_{i j}(x, t)<0,\end{cases} \\
& \underline{g}_{i j}(t, \alpha)= \begin{cases}k_{i j}(x, t) \underline{u}_{j}(t, \alpha), & k_{i j}(x, t) \geq 0 \\
k_{i j}(x, t) \bar{u}_{j}(t, \alpha), & k_{i j}(x, t)<0 .\end{cases}
\end{aligned}
$$

\section{Taylor Expansion Method}

This method depends on differentiating the fuzzy integral equation of the second kind $n$ - times; then substitute the Taylor series expansion for the unknown function into the integral equation. As a result, we get a linear system for which the solution of this system yields the unknown Taylor coefficients of the solution functions.
From (2), (3), and (4) we have the following system:

$$
\begin{aligned}
& \underline{u}_{i}(x, \alpha)=\underline{f}_{i}(x, \alpha)+\sum_{j=1}^{i} \lambda_{i, j}\left(\int_{a}^{c_{i, j}} k_{i, j}(x, t) \underline{u}_{j}(t, \alpha) d t\right. \\
& \left.\quad+\int_{c_{i, j}}^{x} k_{i, j}(x, t) \bar{u}_{j}(t, \alpha) d t\right), \\
& \bar{u}_{i}(x, \alpha)=\bar{f}_{i}(x, \alpha)+\sum_{j=1}^{i} \lambda_{i, j}\left(\int_{a}^{c_{i, j}} k_{i, j}(x, t) \bar{u}_{j}(t, \alpha) d t\right. \\
& \left.\quad+\int_{c_{i, j}}^{x} k_{i, j}(x, t) \underline{u}_{j}(t, \alpha) d t\right) .
\end{aligned}
$$

We seek the solution of system (5) in the form of

$$
\begin{aligned}
\underline{u}_{j, s}(t, \alpha) & =\sum_{r=0}^{s}\left(\left.\frac{1}{r !} \frac{\partial^{(r)} \underline{u}_{j s}(x, \alpha)}{r}\right|_{x=z}(t-z)^{r}\right), \\
\bar{u}_{j, s}(t, \alpha) & =\sum_{r=0}^{s}\left(\left.\frac{1}{r !} \frac{\partial^{(r)} \bar{u}_{j s}(x, \alpha)}{\partial x^{r}}\right|_{x=z}(t-z)^{r}\right) \\
a & \leq x, z \leq b,
\end{aligned}
$$

for $j=1, \ldots, m$, which are the Taylor expansion of degree $s$ at $x=z$ for the unknown functions $\underline{u}_{j s}(x, \alpha)$ and $\bar{u}_{j s}(x, \alpha)$, respectively.

To obtain the solution in the form of expression (6) we find the $n$th derivative of each equation in system (5) with respect to $x$ by using the Leibniz rule, $(n=0,1, \ldots, s)$ and obtain [21]

$$
\begin{aligned}
& \frac{\partial^{(n)} \underline{u}_{i s}(x, \alpha)}{\partial x^{n}}=\frac{\partial^{(n)} \underline{f}_{i}(x, \alpha)}{\partial x^{n}} \\
& +\sum_{j=1}^{m} \lambda_{i, j}\left\{\int_{a}^{c_{i, j}} \frac{\partial^{(n)} k_{i, j}(x, t)}{\partial x^{n}} \underline{u}_{j s}(t, \alpha) d t\right. \\
& +\sum_{l=0}^{n-1}\left(\left.\frac{\partial^{(l)} k_{i, j}(x, t)}{\partial x^{l}}\right|_{t=x} \bar{u}_{j s}(x, \alpha)\right)^{(n-l-1)} \\
& \left.+\int_{c_{i, j}}^{x} \frac{\partial^{(n)} k_{i, j}(x, t)}{\partial x^{n}} \bar{u}_{j s}(t, \alpha) d t\right\}, \\
& \frac{\partial^{(n)} \bar{u}_{j s}(x, \alpha)}{\partial x^{n}}=\frac{\partial^{(n)} \bar{f}_{i}(x, \alpha)}{\partial x^{n}} \\
& +\sum_{j=1}^{m} \lambda_{i, j}\left\{\int_{a}^{c_{i, j}} \frac{\partial^{(n)} k_{i, j}(x, t)}{\partial x^{n}} \bar{u}_{j s}(t, \alpha) d t\right.
\end{aligned}
$$




$$
\begin{aligned}
& +\sum_{l=0}^{n-1}\left(\left.\frac{\partial^{(l)} k_{i, j}(x, t)}{\partial x^{l}}\right|_{t=x} \bar{u}_{j s}(x, \alpha)\right)^{(n-l-1)} \\
& \left.+\int_{c_{i, j}}^{x} \frac{\partial^{(n)} k_{i, j}(x, t)}{\partial x^{n}} \underline{u}_{j s}(t, \alpha) d t\right\}
\end{aligned}
$$

for $n=0,1, \ldots, s$ and $i=1,2, \ldots, m$.

Using the Leibniz rule which is dealing with differentiation of product of functions, system (7) becomes

$$
\begin{aligned}
& \frac{\partial^{(n)} \underline{u}_{i s}(x, \alpha)}{\partial x^{n}}=\frac{\partial^{(n)} \underline{f}_{i}(x, \alpha)}{\partial x^{n}}+\sum_{j=1}^{m} \lambda_{i, j} \\
& \cdot\left\{\int_{a}^{c_{i, j}} \frac{\partial^{(n)} k_{i, j}(x, t)}{\partial x^{n}} \underline{u}_{j s}(t, \alpha) d t\right. \\
& +\sum_{r=0}^{n-1} \sum_{l=0}^{n-r-1}\left(\begin{array}{c}
n-l-1 \\
r
\end{array}\right)\left(\left.\frac{\partial^{(l)} k_{i, j}(x, t)}{\partial x^{l}}\right|_{t=x}\right)^{(n-l-r-1)} \\
& \left.\cdot\left(\bar{u}_{j s}(x, \alpha)\right)^{(r)}+\int_{c_{i, j}}^{x} \frac{\partial^{(n)} k_{i, j}(x, t)}{\partial x^{n}} \bar{u}_{j s}(t, \alpha) d t\right\}, \\
& \frac{\partial^{(n)} \bar{u}_{i s}(x, \alpha)}{\partial x^{n}}=\frac{\partial^{(n)} \bar{f}_{i}(x, \alpha)}{\partial x^{n}}+\sum_{j=1}^{m} \lambda_{i, j} \\
& \cdot\left\{\int_{a}^{c_{i, j}} \frac{\partial^{(n)} k_{i, j}(x, t)}{\partial x^{n}} \bar{u}_{j s}(t, \alpha) d t\right. \\
& \quad+\sum_{r=0}^{n-1} \sum_{l=0}^{n-r-1}\left(\begin{array}{c}
n-l-1 \\
r
\end{array}\right)\left(\left.\frac{\partial^{(l)} k_{i, j}(x, t)}{\partial x^{l}}\right|_{t=x}\right)^{(n-l-r-1)} \\
& \left.\cdot\left(\underline{u}_{j s}(x, \alpha)\right)^{(r)}+\int_{c_{i, j}}^{x} \frac{\partial^{(n)} k_{i, j}(x, t)}{\partial x^{n}} \underline{u}_{j s}(t, \alpha) d t\right\} .
\end{aligned}
$$

Our objective is to determine the coefficients $\underline{u}_{j s}{ }^{(n)}(z, \alpha)$ and $\bar{u}_{j s}{ }^{(n)}(z, \alpha)$, for $n=0, \ldots, s$ and $j=1, \ldots, m$ in system (7); thus we expand $\underline{u}_{j s}(t, \alpha)$ and $\bar{u}_{j s}(t, \alpha)$ in Taylor's series at arbitrary point $z: a \leq z \leq b$

$$
\begin{aligned}
& \underline{u}_{j, s}(t, \alpha)=\sum_{r=0}^{s}\left(\left.\frac{1}{r !} \frac{\partial^{(r)} \underline{u}_{j, s}(x, \alpha)}{\partial x^{r}}\right|_{x=z} \cdot(t-z)^{r}\right), \\
& \bar{u}_{j, s}(t, \alpha)=\sum_{r=0}^{s}\left(\left.\frac{1}{r !} \frac{\partial^{(r)} \bar{u}_{j, s}(x, \alpha)}{\partial x^{r}}\right|_{x=z} \cdot(t-z)^{r}\right),
\end{aligned}
$$$$
a \leq x, z \leq b,
$$

$0 \leq \alpha \leq 1$, for $j=1, \ldots, m$.
Substituting (9) into (8) gives

$$
\begin{gathered}
\underline{u}_{i, s}^{(n)}(z, \alpha)=\underline{f}_{i}^{(n)}(z, \alpha)+\sum_{j=1}^{m}\left\{\sum_{r=0}^{n-1} D_{n, r}^{(i, j)} \bar{u}_{j S}^{(r)}(z, \alpha)\right. \\
\left.+\sum_{r=0}^{s} E_{n, r}^{(i, j)} \underline{u}_{j S}^{(r)}(z, \alpha)+\sum_{r=0}^{s}{E^{\prime}}_{n, r}^{(i, j)} \bar{u}_{j S}^{(r)}(z, \alpha)\right\}, \\
\bar{u}_{i, s}^{(n)}(z, \alpha)=\bar{f}_{i}^{(n)}(z, \alpha)+\sum_{j=1}^{m}\left\{\sum_{r=0}^{n-1} D_{n, r}^{(i, j)} \underline{u}_{j S}^{(r)}(z, \alpha)\right. \\
\left.+\sum_{r=0}^{S} E_{n, r}^{(i, j)} \bar{u}_{j S}^{(r)}(z, \alpha)+\sum_{r=0}^{s}{E^{\prime}}_{n, r}^{(i, j)} \underline{u}_{j S}^{(r)}(z, \alpha)\right\},
\end{gathered}
$$

where

$$
\begin{aligned}
& E_{n, r}^{(i, j)}=\left.\frac{\lambda_{i, j}}{r !} \int_{a}^{c_{i, j}} \frac{\partial^{(n)} k_{i, j}}{\partial x^{n}}(x, t)\right|_{x=z}(t-z)^{r} d t, \\
& E_{n, r}^{(i, j)}=\left.\frac{\lambda_{i, j}}{r !} \int_{c_{i, j}}^{Z} \frac{\partial^{(n)} k_{i, j}}{\partial x^{n}}(x, t)\right|_{x=z}(t-z)^{r} d t, \\
& D_{n, r}^{(i, j)} \\
& =\lambda_{i, j} \sum_{r=0}^{n-l}\left(\begin{array}{c}
n-l-1 \\
r
\end{array}\right)\left(\left.\frac{\partial^{(r)} k_{i, j}}{\partial x^{r}}(x, t)\right|_{x=z}\right)^{(n-l-r-1)}, \\
& \text { for } n=0, \sum_{j=1}^{m} \sum_{r=0}^{n-1} D_{n, r}^{(i, j)} \bar{u}_{j S}^{(r)}(z, \alpha)=0, \text { for } n \leq r, \text { we have } \\
& D_{n, r}^{(i, j)}=0 \text {, and } n, r=0,1, \ldots, s .
\end{aligned}
$$

Consequently, (10) can be written in the matrix form:

$$
(D+E) U=\mathscr{F},
$$

where

$$
\begin{aligned}
F & =\left[-\underline{f}_{1}(z, \alpha), \ldots,-\left.\underline{f}_{1}^{(S)}(x, \alpha)\right|_{x=z},-\bar{f}_{1}(z, \alpha), \ldots,\right. \\
& -\left.\bar{f}_{1}^{(S)}(x, \alpha)\right|_{x=z}, \ldots,-\underline{f}_{m}(z, \alpha), \ldots,-\left.\underline{f}_{m}^{(S)}(x, \alpha)\right|_{x=z}, \\
& \left.-\bar{f}_{m}(z, \alpha), \ldots,-\left.\bar{f}_{m}^{(S)}(x, \alpha)\right|_{x=z}\right]^{t},
\end{aligned}
$$




$$
\begin{aligned}
U & =\left[\underline{u}_{1 S}(z, \alpha), \ldots,\left.\underline{u}_{1 S}^{(S)}(x, \alpha)\right|_{x=z}, \bar{u}_{1 S}(z, \alpha), \ldots,\right. \\
& \left.\bar{u}_{1 S}^{(S)}(x, \alpha)\right|_{x=z}, \ldots, \underline{u}_{m S}(z, \alpha), \ldots,\left.\underline{u}_{m S}^{(S)}(x, \alpha)\right|_{x=z}, \\
& \left.\bar{u}_{m S}(z, \alpha), \ldots,\left.\bar{u}_{m S}^{(S)}(x, \alpha)\right|_{x=z}\right]^{t}, \\
D & =\left[\begin{array}{ccc}
D^{(1,1)} & \cdots & D^{(1, m)} \\
\vdots & \ddots & \vdots \\
D^{(m, 1)} & \cdots & D^{(m, m)}
\end{array}\right], \\
E & =\left[\begin{array}{ccc}
E^{(1,1)} & \cdots & E^{(1, m)} \\
\vdots & \ddots & \vdots \\
E^{(m, 1)} & \cdots & E^{(m, m)}
\end{array}\right] .
\end{aligned}
$$

The Parochial matrices $D^{(i, j)}$ are defined by the following elements (see [14]):

$$
\begin{aligned}
& D^{(i, j)}=\left[\begin{array}{ll}
D_{1,1}^{(i, j)} & D_{1,2}^{(i, j)} \\
D_{2,1}^{(i, j)} & D_{2,2}^{(i, j)}
\end{array}\right], \\
& E^{(i, j)}=\left[\begin{array}{ll}
E_{1,1}^{(i, j)} & E_{1,2}^{(i, j)} \\
E_{2,1}^{(i, j)} & E_{2,2}^{(i, j)}
\end{array}\right],
\end{aligned}
$$

$$
i, j=1, \ldots, m
$$

$E_{1,1}^{(i, j)}=E_{2,2}^{(i, j)}$

$$
=\left[\begin{array}{ccccc}
e_{0,0}^{(i, j)}-1 & e_{0,1}^{(i, j)} & \cdots & e_{0, s-1}^{(i, j)} & e_{0, s}^{(i, j)} \\
e_{1,0}^{(i, j)} & e_{1,1}^{(i, j)}-1 & \cdots & e_{1, s-1}^{(i, j)} & e_{1, s}^{(i, j)} \\
\vdots & \vdots & \ddots & \vdots & \vdots \\
e_{s-1,1}^{(i, j)} & e_{s-1,1}^{(i, j)} & \cdots & e_{s-1, s-1}^{(i, j)}-1 & e_{s-1, s}^{(i, j)} \\
e_{s, 0}^{(i, j)} & e_{s, 1}^{(i, j)} & \cdots & e_{s, s-1}^{(i, j)} & e_{s, s}^{(i, j)}-1
\end{array}\right],
$$

$E_{1,2}^{(i, j)}=E_{2,1}^{(i, j)}$

$$
=\left[\begin{array}{ccccc}
e_{0,0}^{\prime(i, j)}-1 & e_{0,1}^{\prime(i, j)} & \cdots & e_{0, s-1}^{\prime(i, j)} & e_{0, s}^{\prime(i, j)} \\
e^{\prime(i, j)} & e^{\prime(i, j)}-1 & \cdots & e^{\prime(i, j)} & e_{1, s-1}^{\prime(i, j)} \\
\vdots & \vdots & \ddots & \vdots & \vdots \\
e_{s-1,0}^{\prime(i, j)} & e_{s-1,1}^{\prime(i, j)} & \cdots & e_{s-1, s-1}^{\prime(i, j)}-1 & e_{s-1, s}^{\prime(i, j)} \\
e_{s, 0}^{\prime(i, j)} & e_{s, 1}^{\prime(i, j)} & \cdots & e_{s, s-1}^{\prime(i, j)} & e_{s, s}^{\prime(i, j)}-1
\end{array}\right],
$$

$$
\begin{aligned}
D_{1,2}^{(i, j)}=D_{2,1}^{(i, j)}= & {\left[\begin{array}{ccccc}
0 & 0 & \cdots & 0 & 0 \\
d^{\prime(i, j)} & 0 & \cdots & 0 & 0 \\
\vdots & \vdots & \ddots & \vdots & \vdots \\
d^{\prime(i, j)} & d_{s-1,0}^{\prime(i, j)} & \cdots & 0 & 0 \\
d^{\prime(i, j)} & d_{s, 1}^{\prime(i, j)} & \cdots & d_{s, s-1}^{\prime(i, j)} & 0
\end{array}\right], } \\
D_{1,1}^{(i, j)}=D_{2,2}^{(i, j)} & =\left[\begin{array}{ccccc}
0 & 0 & \cdots & 0 & 0 \\
0 & 0 & \cdots & 0 & 0 \\
\vdots & \vdots & \ddots & \vdots & \vdots \\
0 & 0 & \cdots & 0 & 0 \\
0 & 0 & \cdots & 0 & 0
\end{array}\right]_{(s+1) \times(s+1)}
\end{aligned}
$$

3.1. Convergence Analysis. One can show that the above numerical method converges to the exact solution of the fuzzy system (2) (see [14] for more details).

Theorem 2 (see [14]). Let the kernel be bounded and belong to $G:[a, b] *[a, b]$ and $\underline{u}_{j, s}(x, \alpha), \bar{u}_{j, s}(x, \alpha)$ (for $\left.j=1, \ldots, m\right)$ are Taylor polynomials of degree $s$, and their coefficients are computed by solving linear system (12); then these polynomials converge to the exact solution of fuzzy system (2), when $s \rightarrow$ $+\infty$.

\section{Variational Iteration Method}

This method provides a sequence of functions, which converges to the exact solution of the problem and is based on the use of Lagrange multipliers for identification of optimal value of a parameter in a functional. Consider the following general nonlinear system [22]:

$$
L[u(x)]+N[u(t)]=h(x),
$$

where $L$ is a linear operator, $N$ is a nonlinear operator, and $h(x)$ is a given continuous function. According to the variational iteration method, we can construct the following correction functional:

$$
\begin{aligned}
u_{p+1}(x) & \\
= & u_{p}(x) \\
& +\int_{0}^{x} \lambda(z)\left[L\left[u_{p}(z)\right]+N\left[\widetilde{u}_{p}(z)\right]-h(z)\right] d z,
\end{aligned}
$$

where $p=0,1,3, \ldots, \lambda$ is Lagrange multiplier which can be identified optimally via variational theory, $u_{p}$ is the $p$ th approximate solution, and $\widetilde{u}_{p}$ is a restricted variation (i.e., $\delta \widetilde{u}_{p}=0$ ).

Next, we take the partial derivative to both sides of the Volterra integral equation (1) with respect to $x$, and we get

$$
u^{\prime}(x)=f^{\prime}(x)+\frac{d}{d x} \int_{a}^{x} k(x, t) u(t) d t .
$$


Take

$$
\frac{d}{d x} \int_{a}^{x} k(x, t) u(t) d t
$$

as a restricted variation and use the variational iteration method in direction $x$, then we obtain the following iteration sequence:

$$
\begin{gathered}
u_{p+1}(x)=u_{p}(x)+\int_{0}^{x} \lambda(z)\left[u_{p}^{\prime}(z)-f^{\prime}(Z)\right. \\
\left.-\frac{d}{d z} \int_{a}^{z} k(z, t) u_{p}(t) d t\right] d z,
\end{gathered}
$$

and finally we calculate variation with respect to $u_{p}$; notice that $\delta u_{p}(0)=0$ yields

$$
\delta u_{p+1}=\delta u_{p}+\left.\lambda \delta u_{p}\right|_{z=x}-\int_{0}^{x} \lambda^{\prime}(z) \delta u_{p} d z=0
$$

therefore, we get the following stationary conditions:

$$
\begin{aligned}
\left.\lambda^{\prime}(z)\right|_{z=x} & =0, \\
1+\left.\lambda(z)\right|_{z=x} & =0 ;
\end{aligned}
$$

hence, the Lagrange multiplier can be identified: $\lambda(z)=-1$.

If we substitute the value of the Lagrange multiplier into (19), we obtain the following iteration formula:

$$
\begin{gathered}
u_{p+1}(x)=u_{p}(x)-\int_{0}^{x}\left[u_{p}^{\prime}(z)-f^{\prime}(z)\right. \\
\left.-\frac{d}{d z} \int_{a}^{z} k(z, t) u_{p}(t) d t\right] d z .
\end{gathered}
$$

We apply the variational iteration method on fuzzy Volterra integral equation of the second kind:

$$
\begin{aligned}
& \underline{u}(x, \alpha)=\underline{f}(x, \alpha)+\lambda \int_{a}^{x} \underline{k(x, t) u(t, \alpha)} d t \\
& \bar{u}(x, \alpha)=\bar{f}(x, \alpha)+\lambda \int_{a}^{x} \overline{k(x, t) u(t, \alpha)} d t .
\end{aligned}
$$

Suppose that the kernel $k(x, t)>0$ for $a \leq t \leq c$ and $k(x, t)<$ 0 for $c \leq t \leq x$, and then (23) becomes [12]

$$
\begin{aligned}
\underline{u}(x, \alpha)= & \underline{f}(x, \alpha)+\lambda \int_{a}^{c} k(x, t) \underline{u}(t, \alpha) d t \\
& +\lambda \int_{c}^{x} k(x, t) \bar{u}(t, \alpha) d t, \\
\bar{u}(x, \alpha)= & \bar{f}(x, \alpha)+\lambda \int_{a}^{c} k(x, t) \bar{u}(t, \alpha) d t \\
& +\lambda \int_{c}^{x} k(x, t) \underline{u}(t, \alpha) d t,
\end{aligned}
$$

for each $0 \leq \alpha \leq 1$ and $a \leq x \leq b$.
Now, using the variational iteration method and (22), we get the following iteration formulas:

$$
\begin{gathered}
\underline{u}_{p+1}(x, \alpha)=\underline{u}_{p}(x, \alpha) \int_{0}^{x}\left[\underline{u}_{p}^{\prime}(z, \alpha)-\underline{f}^{\prime}(z, \alpha)\right. \\
-\int_{a}^{C} \frac{\partial k(z, t)}{\partial z} \underline{u}_{p}(t, \alpha) d t-k(z, z) \bar{u}_{p}(z, \alpha) \\
\left.-\int_{c}^{z} \frac{\partial k(z, t)}{\partial z}(t, \alpha) \bar{u}_{p}(t, \alpha) d t\right] d z \\
\bar{u}_{p+1}(x, \alpha)=\bar{u}_{p}(x, \alpha)-\int_{0}^{x}\left[\bar{u}_{p}^{\prime}(z, \alpha)-\bar{f}^{\prime}(z, \alpha)\right. \\
-\int_{a}^{C} \frac{\partial k(z, t)}{\partial z} \bar{u}_{p}(t, \alpha) d t-k(z, z) u(z, \alpha) \\
\left.-\int_{c}^{z} \frac{\partial k(z, t)}{\partial z}(t, \alpha) \underline{u}_{k}(t, \alpha) d t\right] d z,
\end{gathered}
$$

where $p=0,1,2, \ldots$

In virtue of (25), we can find a solution of (24) and hence obtain a fuzzy solution of the linear fuzzy Volterra integral equation of the second kind.

\section{Numerical Examples and Results}

In this section, in order to examine the accuracy of the proposed methods, we have chosen one example of linear fuzzy integral equation of the second kind. Moreover, the numerical results will be compared with the exact solution.

Example 3 (Taylor expansion method). Consider the following fuzzy linear Volterra integral equations:

$$
\begin{aligned}
& \underline{u}(x, \alpha)=(\alpha) \cot (x)+\int_{0}^{x} e^{x-t} \underline{u}(t, \alpha) d t, \\
& \bar{u}(x, \alpha)=(2-\alpha) \cot (x)+\int_{0}^{x} e^{x-t} \bar{u}(t, \alpha) d t, \\
& \quad 0 \leq \alpha \leq 1,0 \leq x \leq \frac{\pi}{4} .
\end{aligned}
$$

The analytical solution of the above equation is given as

$$
\begin{aligned}
& \underline{u}(x, \alpha)=(\alpha)\left(\frac{3}{5} \cos (x)+\frac{1}{5} \sin (x)+\frac{2}{5} e^{2 x}\right), \\
& \bar{u}(x, \alpha)=(2-\alpha)\left(\frac{3}{5} \cos (x)+\frac{1}{5} \sin (x)+\frac{2}{5} e^{2 x}\right), \\
& 0 \leq \alpha \leq 1 .
\end{aligned}
$$

Expand the unknown functions in Taylor series at $z=\pi / 12$ and implement the following algorithm.

\section{Algorithm 4.}

(1) Input $a, b, z, m, \lambda, k_{i, j}(x, t), \underline{f_{i}}(x, \alpha), \bar{f}_{i}(x, \alpha)$.

(2) Input the Taylor expansion degree $(s)$. 
(3) Calculate

$$
\begin{gathered}
\frac{\partial^{(n)} k_{i, j}(x, \alpha)}{\partial x^{n}} \\
\frac{\partial^{(n)} \underline{f}_{i}(x, \alpha)}{\partial x^{n}}, \\
\frac{\partial^{(n)} \bar{f}_{i}(x, \alpha)}{\partial x^{n}}
\end{gathered}
$$$$
n=0,1, \ldots, s \text {. }
$$

(4) Calculate

$$
\begin{aligned}
e_{n+1, r+1}^{(i, j)}=\frac{\lambda_{i, j}}{r !} \int_{a}^{c_{i, j}} \frac{\partial^{r} k_{i, j}}{\partial x^{r}}(x, t) \cdot(t-z)^{r} d t & \\
& i, j=1, \ldots, m .
\end{aligned}
$$

(5) Calculate

$$
\begin{aligned}
e_{n+1, r+1}^{\prime(i, j)}=\frac{\lambda_{i, j}}{r !} \int_{a}^{c_{i, j}} \frac{\partial^{r} k_{i, j}}{\partial x^{r}}(x, t) \cdot(t-z)^{r} d t & \\
& i, j=1, \ldots, m .
\end{aligned}
$$

(6) Put

$$
\begin{aligned}
& E_{1,1}^{(i, j)}=E_{2,2}^{(i, j)} \\
& =\left[\begin{array}{ccccc}
e_{1,1}^{(i, j)}-1 & e_{1,2}^{(i, j)} & \cdots & e_{1, s}^{(i, j)} & e_{1, s+1}^{(i, j)} \\
e_{2,1}^{(i, j)} & e_{2,2}^{(i, j)}-1 & \cdots & e_{2, s}^{(i, j)} & e_{2, s+1}^{(i, j)} \\
\vdots & \vdots & \ddots & \vdots & \vdots \\
e_{s, 1}^{(i, j)} & e_{s, 2}^{(i, j)} & \cdots & e_{s, s}^{(i, j)}-1 & e_{s, s+1}^{(i, j)} \\
e_{s+1,1}^{(i, j)} & e_{s+1,2}^{(i, j)} & \cdots & e_{s+1, s}^{(i, j)} & e_{s+1, s+1}^{(i, j)}-1
\end{array}\right]_{(s+1) \times(s+1)}
\end{aligned}
$$

${E^{\prime}}_{1,2}^{(i, j)}={E^{\prime}}_{2,1}^{(i, j)}$

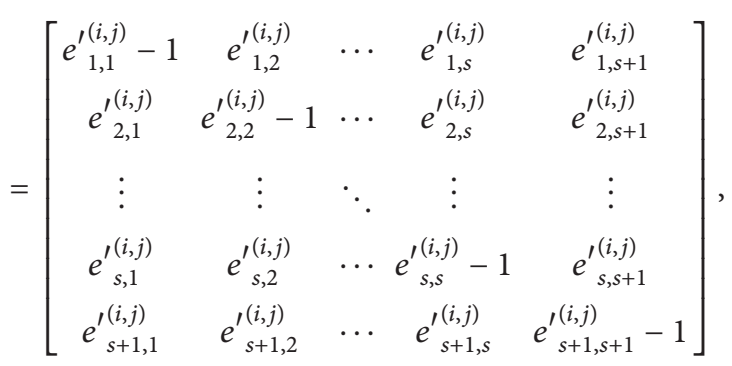

where $i, j=1,2, \ldots, m$.

(8) Calculate

$$
\begin{aligned}
& d_{n+1, l+1}^{\prime} \\
& =\left.\sum_{r=0}^{n-l-1}\left(\begin{array}{c}
n-r-1 \\
n-l-r-1
\end{array}\right)\left(\left.\frac{\partial^{(r)} k_{i, j}(x, t)}{\partial x^{r}}\right|_{t=x}\right)^{(n-l-r-1)}\right|_{x=z} .
\end{aligned}
$$

(9) Put

$$
D_{1,1}^{(i, j)}=D_{2,2}^{(i, j)}=\left[\begin{array}{ccccc}
0 & 0 & \cdots & 0 & 0 \\
0 & 0 & \cdots & 0 & 0 \\
\vdots & \vdots & \ddots & \vdots & \vdots \\
0 & 0 & \cdots & 0 & 0 \\
0 & 0 & \cdots & 0 & 0
\end{array}\right]_{(s+1) \times(s+1)} .
$$

(10) Put

$$
{D^{\prime}}_{1,2}^{(i, j)}=D_{2,1}^{\prime(i, j)}
$$

$$
=\left[\begin{array}{ccccc}
0 & 0 & \cdots & 0 & 0 \\
d_{2,1}^{\prime(i, j)} & 0 & \cdots & 0 & 0 \\
\vdots & \vdots & \ddots & \vdots & \vdots \\
d_{k, 1}^{\prime(i, j)} & d_{k, 2}^{\prime(i, j)} & \cdots & 0 & 0 \\
d_{k+1,1}^{\prime(i, j)} & d_{k+1,2}^{\prime(i, j)} & \cdots & d_{k+1, k}^{\prime(i, j)} & 0
\end{array}\right]_{(s+1) \times(s+1)}
$$

(11) Denote

$$
D^{(i, j)}=\left[\begin{array}{cc}
D_{1,1}^{(i, j)} & D_{1,2}^{(i, j)} \\
D_{2,1}^{(i, j)} & D_{2,2}^{(i, j)}
\end{array}\right] .
$$

(12) Put

$$
\begin{aligned}
\mathscr{F} & =\left[-\underline{f}_{1}(z, \alpha), \ldots,-\underline{f}_{1}^{(k)}(z, \alpha),\right. \\
& -\bar{f}_{1}(z, \alpha), \ldots,-\bar{f}_{1}^{(s)}(z, \alpha), \ldots, \\
& -\underline{f}_{m}(z, \alpha), \ldots,-\underline{f}_{m}^{(s)}(z, \alpha), \\
& \left.-\bar{f}_{m}(z, \alpha), \ldots,-\bar{f}_{m}^{(s)}(z, \alpha)\right]^{t} .
\end{aligned}
$$

(13) Put

$$
\begin{aligned}
u= & {\left[\underline{u}_{1 s}(z, \alpha), \ldots, \underline{u}_{1 s}^{(s)}(z, \alpha), \bar{u}_{1 s}(z, \alpha), \ldots, \bar{u}_{1 s}^{(s)}(z, \alpha),\right.} \\
& \ldots, \underline{u}_{m s}(z, \alpha), \ldots, \underline{u}_{m s}^{(s)}(z, \alpha), \bar{u}_{m s}(z, \alpha), \ldots, \\
& \left.\bar{u}_{m s}^{(s)}(z, \alpha)\right]^{t} .
\end{aligned}
$$

(14) Solve the following linear system $(E+D) u=\mathscr{F}$.

(15) Estimate $\underline{u}(z, \alpha), \bar{u}(z, \alpha)$ by computing Taylor expansion for $u$

$$
\begin{aligned}
& \underline{u}_{j, s}=\sum_{r=0}^{s}\left(\left.\frac{1}{r !} \frac{\partial^{(r)} \underline{u}_{j}(x, \alpha)}{\partial x^{r}}\right|_{x=z}(x-z)^{r}\right) \\
& u_{j, s}=\sum_{r=0}^{s}\left(\left.\frac{1}{r !} \frac{\partial^{(r)} \bar{u}_{j}(x, \alpha)}{\partial x^{r}}\right|_{x=z}(x-z)^{r}\right),
\end{aligned}
$$


We get the following results:

$$
\begin{aligned}
& E_{1,1}^{(1,1)}=E_{2,2}^{(1,1)}=\left[\begin{array}{ccccccc}
-.70073 & -.04088 & .00364 & -.00024 & 1.275 \times 10^{-5} & -5.598 \times 10^{-7} \\
.29927 & -1.0409 & .00364 & -.00024 & 1.275 \times 10^{-5} & -5.598 \times 10^{-7} \\
.29927 & -.04088 & -.99636 & -.00024 & 1.275 \times 10^{-5} & -5.598 \times 10^{-7} \\
.29927 & -.04088 & .00364 & -1.0002 & 1.275 \times 10^{-5} & -5.598 \times 10^{-7} \\
.29927 & -.04088 & .00364 & -.00024 & -0.99999 & -5.598 \times 10^{-7} \\
.29927 & -.04088 & .00364 & -.00024 & 1.275 \times 10^{-5} & -1.000
\end{array}\right] \\
& E_{1,2}^{(1,1)}=E_{2,1}^{(1,1)}=\left[\begin{array}{llllll}
0 & 0 & 0 & 0 & 0 & 0 \\
0 & 0 & 0 & 0 & 0 & 0 \\
0 & 0 & 0 & 0 & 0 & 0 \\
0 & 0 & 0 & 0 & 0 & 0 \\
0 & 0 & 0 & 0 & 0 & 0 \\
0 & 0 & 0 & 0 & 0 & 0
\end{array}\right] ;
\end{aligned}
$$

hence

$$
\begin{aligned}
& E=\left[\begin{array}{ll}
E_{1,1}^{(1,1)} & E_{1,2}^{(1,1)} \\
E_{2,1}^{(1,1)} & E_{2,2}^{(1,1)}
\end{array}\right], \\
& D_{1,1}^{(1,1)}=D_{2,2}^{(1,1)}=\left[\begin{array}{llllll}
0 & 0 & 0 & 0 & 0 & 0 \\
1 & 0 & 0 & 0 & 0 & 0 \\
1 & 1 & 0 & 0 & 0 & 0 \\
1 & 1 & 1 & 0 & 0 & 0 \\
1 & 1 & 1 & 1 & 0 & 0 \\
1 & 1 & 1 & 1 & 1 & 0
\end{array}\right] \text {, } \\
& D_{1,2}^{(1,1)}=D_{2,1}^{(1,1)}=\left[\begin{array}{llllll}
0 & 0 & 0 & 0 & 0 & 0 \\
0 & 0 & 0 & 0 & 0 & 0 \\
0 & 0 & 0 & 0 & 0 & 0 \\
0 & 0 & 0 & 0 & 0 & 0 \\
0 & 0 & 0 & 0 & 0 & 0 \\
0 & 0 & 0 & 0 & 0 & 0
\end{array}\right] \text {, } \\
& D=\left[\begin{array}{ll}
D_{1,1}^{(1,1)} & D_{1,2}^{(1,1)} \\
D_{2,1}^{(1,1)} & D_{2,2}^{(1,1)}
\end{array}\right], \\
& \mathscr{F}(z, \alpha)=\left[-\underline{f}(z, \alpha),-\underline{f}^{\prime}(z, \alpha), \underline{-f^{\prime \prime}}(z, \alpha),\right. \\
& -\underline{f}^{\prime \prime \prime}(z, \alpha), \underline{-f^{(4)}}(z, \alpha), \underline{-f^{(5)}}(z, \alpha)-\bar{f}(z, \alpha), \\
& \overline{-f}^{\prime}(z, \alpha),-\bar{f}^{\prime \prime}(z, \alpha),-\bar{f}^{\prime \prime \prime}(z, \alpha),-\bar{f}^{(4)}(z, \alpha) \text {, } \\
& \left.-\bar{f}^{(5)}(z, \alpha)\right]^{t} \text {, }
\end{aligned}
$$

$$
\mathscr{F}=\left[\begin{array}{c}
-\alpha \cos \left(\frac{\pi}{12}\right) \\
\alpha \sin \left(\frac{\pi}{12}\right) \\
\alpha \cos \left(\frac{\pi}{12}\right) \\
-\alpha \sin \left(\frac{\pi}{12}\right) \\
-\alpha \cos \left(\frac{\pi}{12}\right) \\
\alpha \sin \left(\frac{\pi}{12}\right) \\
-(2-\alpha) \cos \left(\frac{\pi}{12}\right) \\
(2-\alpha) \sin \left(\frac{\pi}{12}\right) \\
(2-\alpha) \cos \left(\frac{\pi}{12}\right) \\
-(2-\alpha) \sin \left(\frac{\pi}{12}\right) \\
-(2-\alpha) \cos \left(\frac{\pi}{12}\right) \\
(2-\alpha) \sin \left(\frac{\pi}{12}\right)
\end{array}\right]
$$

Solving the linear system

$$
(D+E) u=\mathscr{F},
$$


we obtain

$$
\begin{aligned}
& u(z, \alpha)=\left[\begin{array}{c}
1.30655494406977 \alpha \\
1.38836501660070 \alpha \\
2.06962325214024 \alpha \\
5.36399137514409 \alpha \\
11.4350895320246 \alpha \\
21.4350895320246 \alpha \\
1.30655494406977(2-\alpha) \\
1.38836501660070(2-\alpha) \\
2.06962325214024(2-\alpha) \\
5.36399137514409(2-\alpha) \\
11.4350895320246(2-\alpha) \\
21.4350895320246(2-\alpha)
\end{array}\right], \\
& \underline{u}(x, \alpha)=\sum_{r=0}^{s}\left(\left.\frac{1}{r !} \frac{\partial^{(r)} \underline{u}_{j}(x, \alpha)}{\partial x^{r}}\right|_{x=z}\left(x-\frac{\pi}{12}\right)^{r}\right), \\
& \bar{u}(x, \alpha)=\sum_{r=0}^{s}\left(\left.\frac{1}{r !} \frac{\partial^{(r)} \bar{u}_{j}(x, \alpha)}{\partial x^{r}}\right|_{x=z}\left(x-\frac{\pi}{12}\right)^{r}\right), \\
& 0 \leq x \leq \frac{\pi}{4}, 0 \leq \alpha \leq 1 .
\end{aligned}
$$

The approximate solution is

$$
\begin{aligned}
\underline{u}(x, \alpha)= & 0.9999816737 \alpha+1.000399233 \alpha x \\
& +0.4962378250+0.5186782119 \alpha x^{3} \\
& +0.2403470047 \alpha x^{4} \\
& +0.1803786182 \alpha x^{5}, \\
\bar{u}(x, \alpha)= & 2.000407015 x+1.738708618 \alpha \\
& +0.3524026447 x^{3}+0.1975243281 x^{4} \\
& +0.1905848255 x^{5}-0.7387269443 \\
& +0.04282267662 \alpha x^{4} \\
& -0.01020620726 \alpha x^{5} \\
& -1.000007782 \alpha x+0.9961625156 x^{2} \\
& -0.4999246903 \alpha x^{2} \\
& +0.1662755673 \alpha x^{3} .
\end{aligned}
$$

Figure 1 compares both the exact and numerical solutions for the fuzzy integral equation (26) using the Taylor expansion method at $x=\pi / 8$. Moreover, Figure 2 shows the absolute error between the exact and numerical solutions of this example.

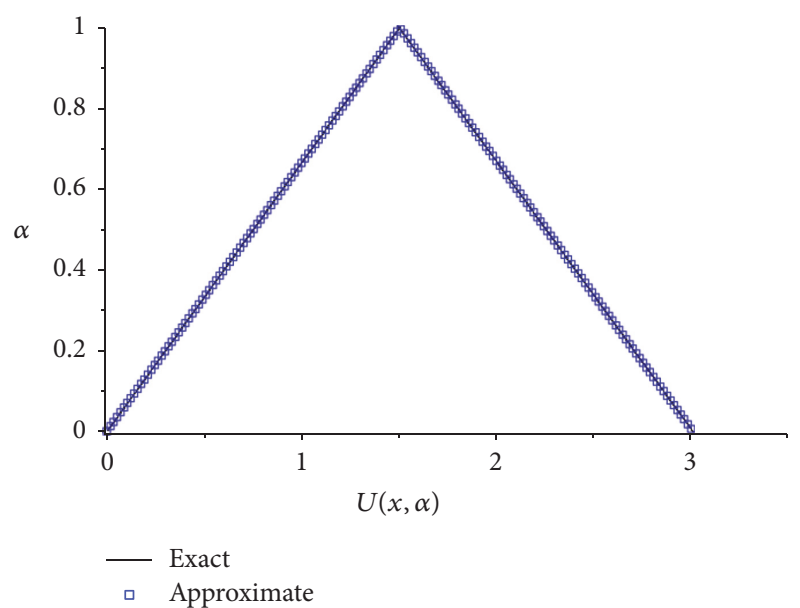

Figure 1: Exact and numerical solutions at $x=\pi / 8$.

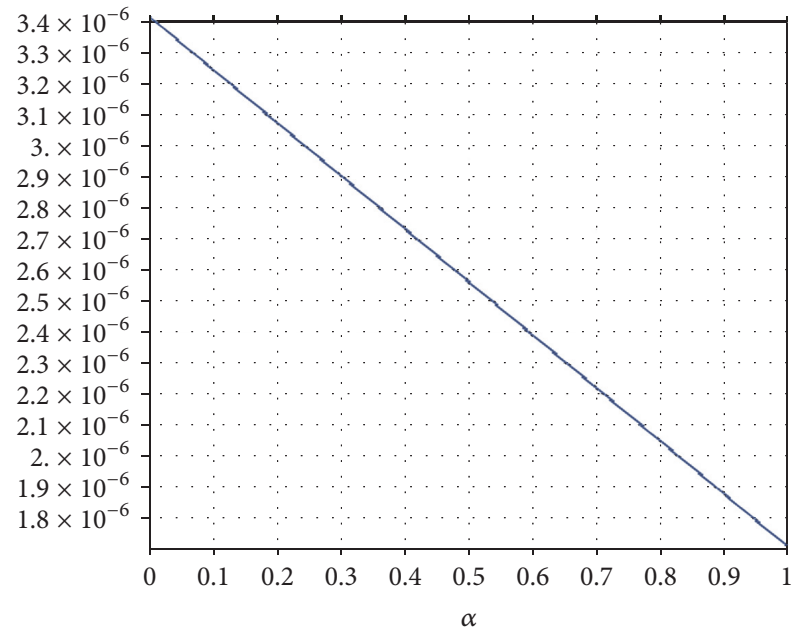

FIGURE 2: Absolute error between the exact and numerical solutions.

Example 5 (variation alliteration method). Consider the linear fuzzy Volterra integral equation (26) with the exact solution (27).

In the view of the variational iteration method, we construct a correction functional in the following form:

$$
\begin{aligned}
& \underline{u}_{p+1}(x, \alpha)=\underline{f}(x, \alpha)+\int_{0}^{x} k(x, t) \underline{u}_{p}(t, \alpha) d t, \\
& \bar{u}_{p+1}(x, \alpha)=\bar{f}(x, \alpha)+\int_{0}^{x} k(x, t) \bar{u}_{p}(t, \alpha) d t,
\end{aligned}
$$

where $p=0,1,2, \ldots, s$.

Start with the initial approximation in (45); that is,

$$
\begin{aligned}
& \underline{u}_{0}(x, \alpha)=(\alpha) \cos x, \\
& \bar{u}_{0}(x, \alpha)=(2-\alpha) \cos x,
\end{aligned}
$$

$$
0 \leq \alpha \leq 1
$$

Then successive approximations $u_{p}(x, \alpha)^{\prime} s$ will be generated. In this example we calculate the 6th order of approximate 
solution using the variational iteration method by MAPLE software. We have carried out the following algorithm.

\section{Algorithm 6.}

(1) Input $a, b, \lambda, k(x, t), s, f(x, \alpha), \bar{f}(x, \alpha)$.

(2) Input $\underline{u}_{0}(x, \alpha), \bar{u}_{0}(x, \alpha)$.

(3) For $p=1$ to $s+1$, compute

$$
\begin{aligned}
& \underline{u}_{p+1}(x, \alpha)=\underline{f}(x, \alpha)+\int_{0}^{x} k(x, t) \underline{u}_{p}(t, \alpha) d t, \\
& \bar{u}_{p+1}(x, \alpha)=\bar{f}(x, \alpha)+\int_{0}^{x} k(x, t) \bar{u}_{p}(t, \alpha) d t .
\end{aligned}
$$

We obtain the following results:

$$
\begin{aligned}
& \underline{u}_{1}(x, \alpha)=\frac{1}{2} \alpha \cos x+\frac{1}{2} \alpha e^{x}+\frac{1}{2} \alpha \sin x \text {, } \\
& \underline{u}_{2}(x, \alpha)=\frac{1}{2} \alpha \cos x+\frac{1}{2} \alpha e^{x}+\frac{1}{2} \alpha e^{x} x, \\
& \underline{u}_{3}(x, \alpha)=\frac{3}{4} \alpha \cos x+\frac{1}{4} \alpha e^{x}+\frac{1}{4} \alpha \sin x+\frac{1}{2} \alpha e^{x} x \\
& +\frac{1}{2} \alpha e^{x} x^{2}, \\
& \underline{u}_{4}(x, \alpha)=\frac{1}{2} \alpha \cos x+\frac{1}{2} \alpha e^{x}+\frac{1}{4} \alpha \sin x+\frac{1}{4} \alpha e^{x} x \\
& +\frac{1}{4} \alpha e^{x} x^{2}+\frac{1}{12} \alpha e^{x} x^{3} \\
& \underline{u}_{5}(x, \alpha)=\frac{5}{8} \alpha \cos x+\frac{3}{8} \alpha e^{x}+\frac{1}{8} \alpha \sin x+\frac{1}{2} \alpha e^{x} x \\
& +\frac{1}{8} \alpha e^{x} x^{2}+\frac{1}{12} \alpha e^{x} x^{3}+\frac{1}{48} \alpha e^{x} x^{4}, \\
& \underline{u}_{6}(x, \alpha)=\frac{5}{8} \alpha \cos x+\frac{3}{8} \alpha e^{x}+\frac{1}{4} \alpha \sin x+\frac{3}{8} \alpha e^{x} x \\
& +\frac{1}{4} \alpha e^{x} x^{2}+\frac{1}{24} \alpha e^{x} x^{3}+\frac{1}{48} \alpha e^{x} x^{4} \\
& +\frac{1}{240} \alpha e^{x} x^{5} \\
& \bar{u}_{1}(x, \alpha)=(2-\alpha) \cos x+e^{x}-\frac{1}{2} \alpha e^{x}-\cos x+\sin x \\
& +\frac{1}{2} \alpha \cos x-\frac{1}{2} \alpha \sin x, \\
& \bar{u}_{2}(x, \alpha)=(2-\alpha) \cos x+e^{x}-\frac{1}{2} \alpha e^{x}-\cos x \\
& +\frac{1}{2} \alpha \cos x+e^{x} x-\frac{1}{2} \alpha e^{x} x,
\end{aligned}
$$

$$
\begin{aligned}
\bar{u}_{3}(x, \alpha)= & (2-\alpha) \cos x+\frac{1}{2} e^{x}-\frac{1}{4} \alpha e^{x}-\frac{1}{2} \cos x \\
& +\frac{1}{2} \sin x+\frac{1}{4} \alpha \cos x e-\frac{1}{4} \alpha \sin x+e^{x} x \\
& -\frac{1}{2} \alpha e^{x} x+\frac{1}{2} e^{x} x^{2}-\frac{1}{4} \alpha e^{x} x^{2}, \\
\bar{u}_{4}(x, \alpha)= & (2-\alpha) \cos x+e^{x}-\frac{1}{2} \alpha e^{x}-\frac{1}{12} \alpha e^{x} x^{3} \\
& +\frac{1}{2} e^{x} x+\frac{1}{2} e^{x} x^{2}+\frac{1}{6} e^{x} x^{3}-\cos x \\
& +\frac{1}{2} \sin x-\frac{1}{4} \alpha e^{x} x-\frac{1}{4} \alpha e^{x} x^{2} \\
& +\frac{1}{2} \alpha \cos x-\frac{1}{4} \alpha \sin x,
\end{aligned}
$$

$$
\begin{aligned}
\bar{u}_{5}(x, \alpha)= & (2-\alpha) \cos x+\frac{3}{4} e^{x}-\frac{3}{8} \alpha e^{x}+\frac{1}{4} e^{x} x^{2} \\
& +\frac{3}{8} \alpha \cos x+\frac{1}{6} e^{x} x^{3} \\
& +\frac{1}{4} \sin x+e^{x} x-\frac{3}{4} \cos x-\frac{1}{48} \alpha e^{x} x^{4} \\
& -\frac{1}{8} \alpha \sin x-\frac{1}{12} \alpha e^{x} x^{3}-\frac{1}{8} \alpha e^{x} x^{2} \\
& -\frac{1}{2} \alpha e^{x} x+\frac{1}{24} e^{x} x^{4},
\end{aligned}
$$$$
\bar{u}_{6}(x, \alpha)=(2-\alpha) \cos x+\frac{3}{4} e^{x}-\frac{3}{8} \alpha e^{x}+\frac{1}{2} e^{x} x^{2}
$$$$
+\frac{3}{8} \alpha \cos x+\frac{1}{12} e^{x} x^{3}
$$$$
+\frac{1}{2} \sin x+\frac{3}{4} e^{x} x-\frac{3}{4} \cos x+\frac{1}{120} e^{x} x^{5}
$$$$
-\frac{1}{240} \alpha e^{x} x^{5}-\frac{1}{48} \alpha e^{x} x^{4}-\frac{1}{4} \alpha \sin x
$$$$
-\frac{1}{24} \alpha e^{x} x^{3}-\frac{1}{4} \alpha e^{x} x^{2}-\frac{3}{8} \alpha e^{x} x
$$$$
+\frac{1}{24} e^{x} x^{4} .
$$

Figure 3 shows a comparison between the exact and the numerical solutions for the fuzzy integral equation (26) by the variational iteration method (VIM) at $x=\pi / 8$. Moreover, Figure 4 shows the absolute error between the exact and numerical solutions of this example.

Table 1 shows a comparison between the exact and the numerical solutions for the fuzzy integral equation (26) using the Taylor expansion method and the variational iteration method (VIM) at $x=\pi / 8$. 


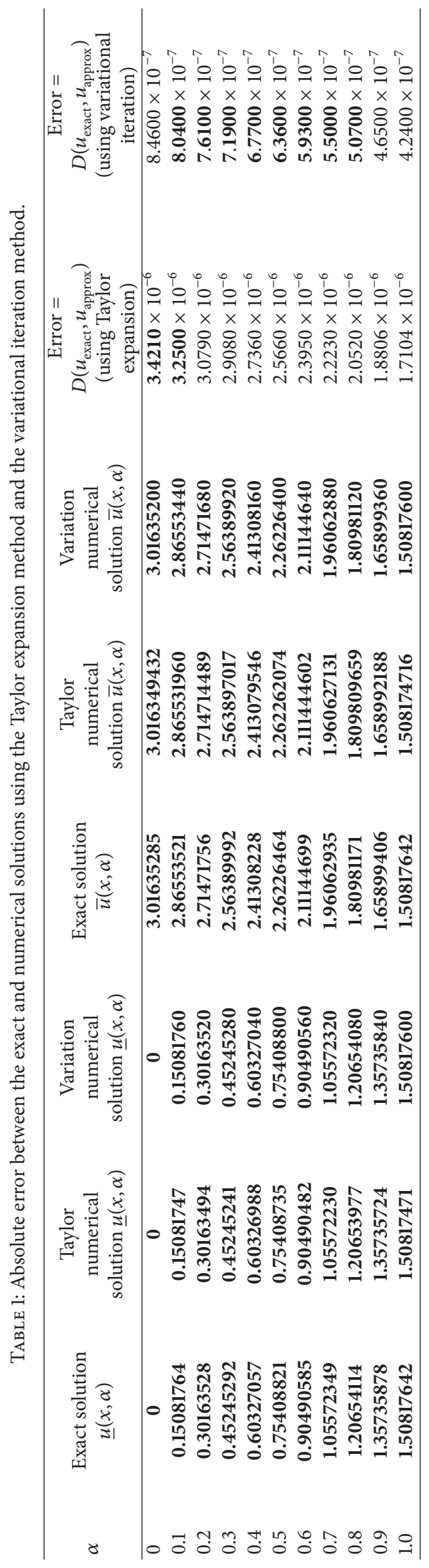




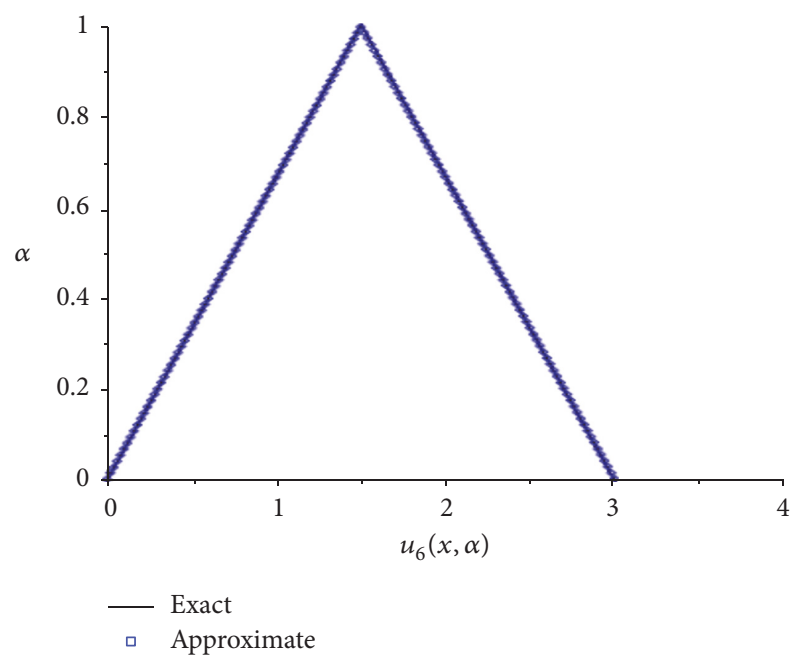

FIGURE 3: Exact and numerical solutions at $x=\pi / 8$.

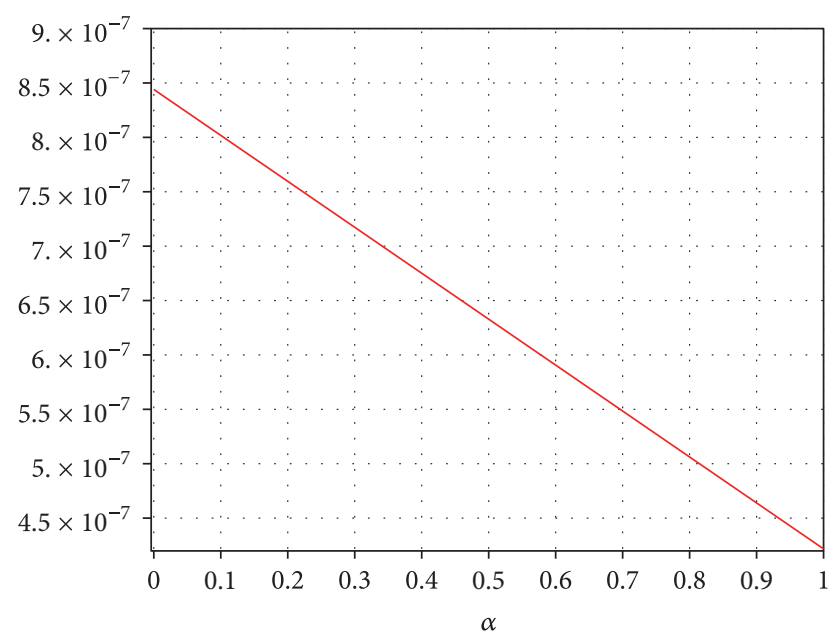

FIgURE 4: Absolute error between the exact and numerical solutions.

\section{Conclusion}

In this article, Taylor expansion and variational iteration methods are proposed to solve a fuzzy linear Volterra integral equation of the second kind. The results of the example show that the convergence and accuracy of both methods were in a good agreement with the analytical solution. According to comparison of numerical results, mentioned in tables and figures, we conclude that the variational iteration method provides more accurate results and therefore is more advantageous.

\section{Conflicts of Interest}

The authors declare that they have no conflicts of interest.

\section{References}

[1] T. Allahviranloo, S. Salahshour, M. Homayoun-Nejad, and D. Baleanu, "General solutions of fully fuzzy linear systems," Abstract and Applied Analysis, vol. 2013, Article ID 593274, 2013.

[2] L. A. Zadeh, "Fuzzy sets," Information and Control, vol. 8, no. 3, pp. 338-353, 1965.

[3] S. S. L. Chang and L. A. Zadeh, "On fuzzy mapping and control," IEEE Transactions on Systems, Man and Cybernetics, vol. 2, no. 1, pp. 30-34, 1972.

[4] L. A. Zadeh, "Linguistic variables, approximate reasoning and dispositions.," Medical Informatics, vol. 8, no. 3, pp. 173-186, 1983.

[5] D. Dubois and H. Prade, "Operations on fuzzy numbers," International Journal of Systems Science. Principles and Applications of Systems and Integration, vol. 9, no. 6, pp. 613-626, 1978.

[6] J. Goetschel and W. Voxman, "Elementary fuzzy calculus," Fuzzy Sets and Systems, vol. 18, no. 1, pp. 31-43, 1986.

[7] O. Kaleva, "Fuzzy differential equations," Fuzzy Sets and Systems, vol. 24, no. 3, pp. 301-317, 1987.

[8] S. Nanda, "On integration of fuzzy mappings," Fuzzy Sets and Systems, vol. 32, no. 1, pp. 95-101, 1989.

[9] M. Friedman, M. Ma, and A. Kandel, "Numerical solutions of fuzzy differential and integral equations," Fuzzy Sets and Systems, vol. 106, no. 1, pp. 35-48, 1999.

[10] F. G. Tricomi, Integral Equations, Dover Publications, New York, NY, USA, 1982.

[11] S. Liao, "Beyond Perturbation: introduction to the homotopy analysis method," in Modern Mechanics and Mathematics, vol. 2, pp. 1-336, Chapman and Hall/CRC Press, Boca Raton, Fla, USA, 2003.

[12] S. Abbasbandy, "Numerical solutions of the integral equations: homotopy perturbation method and Adomian's decomposition method," Applied Mathematics and Computation, vol. 173, no. 1, pp. 493-500, 2006.

[13] E. Babolian, H. Sadeghi Goghary, and S. Abbasbandy, "Numerical solution of linear Fredholm fuzzy integral equations of the second kind by Adomian method," Applied Mathematics and Computation, vol. 161, no. 3, pp. 733-744, 2005.

[14] A. Jafarian, S. Measoomy Nia, and S. Tavan, "A numerical scheme to solve fuzzy linear volterra integral equations system," Journal of Applied Mathematics, vol. 2012, Article ID 216923, 17 pages, 2012.

[15] R. P. Kanwal and K. C. Liu, "A Taylor expansion approach for solving integral equations," International Journal of Mathematical Education in Science and Technology, vol. 20, no. 3, pp. 411414, 1989.

[16] L. Xu, "Variational iteration method for solving integral equations," Computers \& Mathematics with Applications, vol. 54, no. 7-8, pp. 1071-1078, 2007.

[17] M. Amawi, Fuzzy Fredholm Integral Equation of the Second Kind (M. S. Thesis), An-Najah National University, 2014.

[18] M. Amawi and N. Qatanani, "Numerical methods for solving fuzzy Fredholm integral equation of the second kind," International Journal of Applied Mathematics, vol. 28, no. 3, pp. 177-195, 2015.

[19] J. Hamaydi, Analytical and Numerical Methods for Solving Linear Fuzzy Volterra Integral Equation of the Second Kind (M. S. Thesis), Najah National University, 2016.

[20] M. Ghanbari, "Numerical solution of fuzzy linear Volterra integral equations of the second kind by homotopy analysis 
method," International Journal of Industrial Mathematics (IJIM), vol. 2, no. 2, pp. 73-87, 2010.

[21] H. H. Sorkun and S. YalcCinbac, "Approximate solutions of linear Volterra integral equation systems with variable coefficients," Applied Mathematical Modeling, vol. 34, no. 11, pp. 34513464, 2010.

[22] M. Gholami and B. Ghanbari, "Variational iteration method for solving Voltarra and Fredholm integral equations of the second kind," General Mathematics Notes, vol. 2, no. 1, pp. 143-148, 2011. 


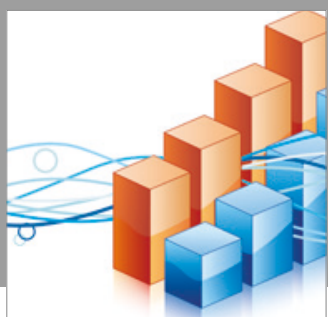

Advances in

Operations Research

vatersals

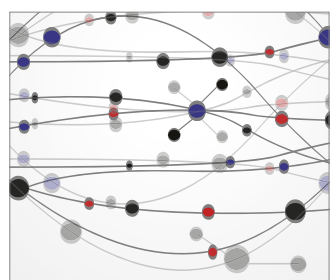

\section{The Scientific} World Journal
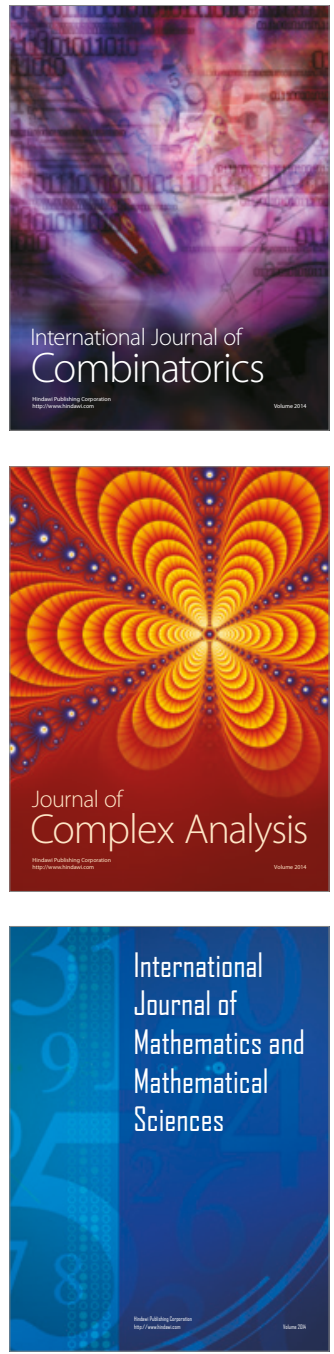
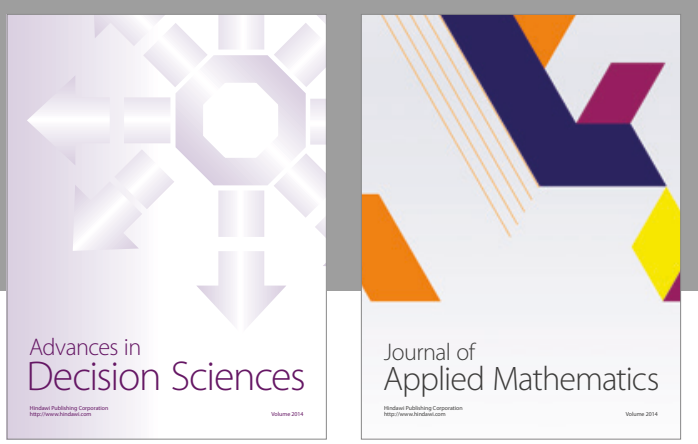

Algebra

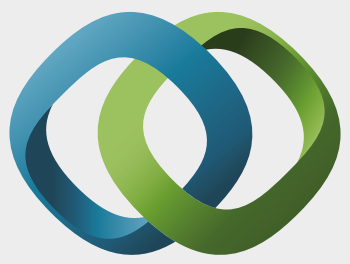

\section{Hindawi}

Submit your manuscripts at

https://www.hindawi.com
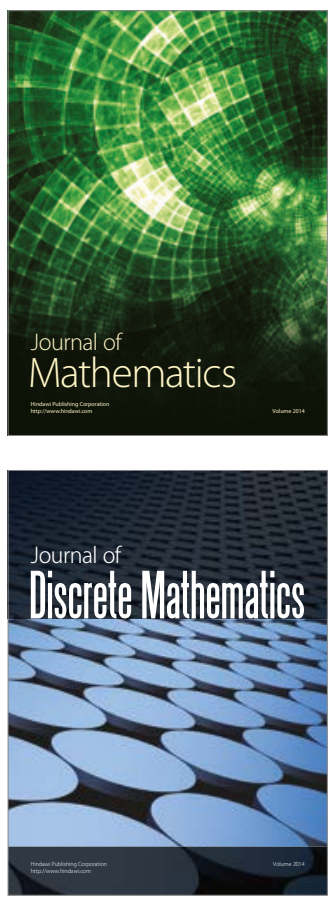

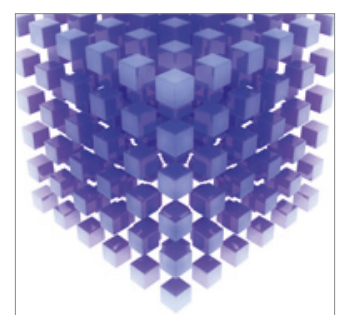

Mathematical Problems in Engineering
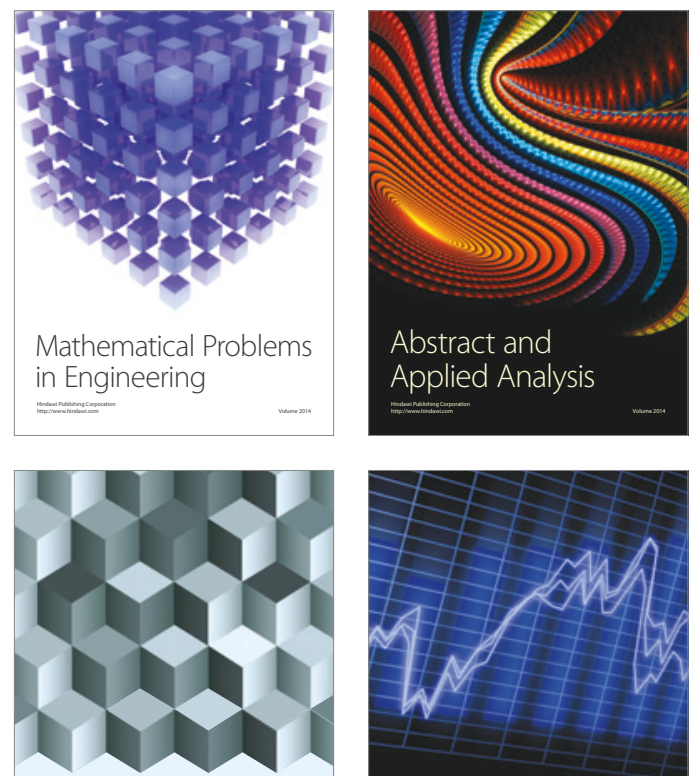

Journal of

Function Spaces

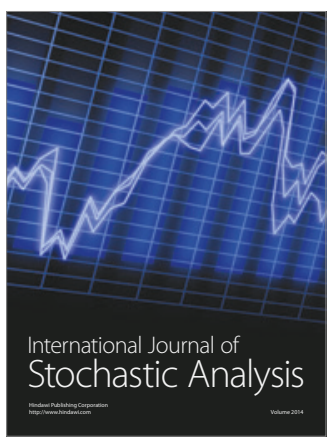

Probability and Statistics
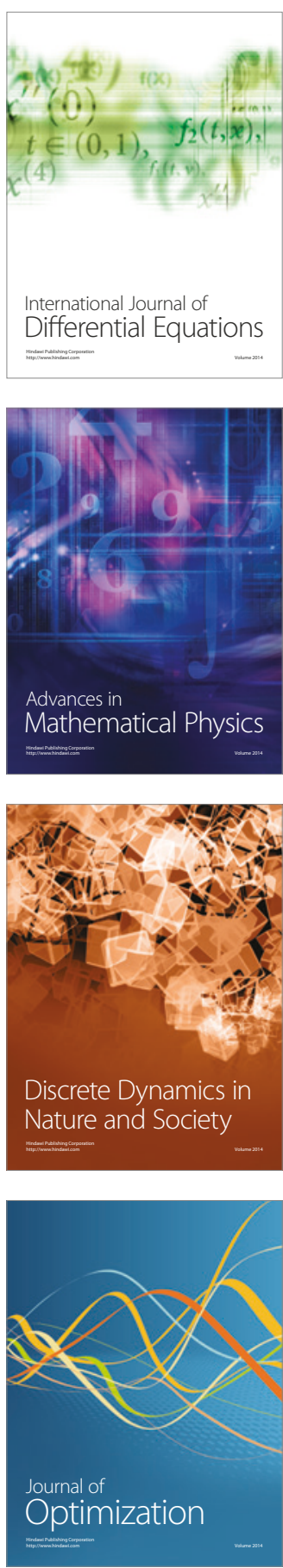